\title{
Electrically Small, Low-Profile, Planar, Huygens Dipole Antenna with Quad-Polarization Diversity
}

\author{
Ming-Chun Tang, Senior Member, IEEE, Zhentian Wu, Ting Shi, Student Member, IEEE, and Richard \\ W. Ziolkowski, Fellow, IEEE
}

\begin{abstract}
An electrically small, low-profile, planar, Huygens dipole antenna with four reconfigurable polarization states is presented. The design incorporates both electric and magnetic near-field resonant parasitic (NFRP) elements and a reconfigurable driven element. The four polarization states include two orthogonal linear (LP) and two circular polarization (LHCP and RHCP) states. A $1.5 \mathrm{GHz}$ prototype was fabricated (partially with 3D additive manufacturing), assembled, and tested. The measured results, in good agreement with their simulated values, demonstrate that even with its simple configuration, electrically small size $(k a=0.944)$, and low-profile height $\left(0.0449 \lambda_{0}\right)$, this reconfigurable Huygens antenna possesses stable broadside radiation performance in all of its four polarization states. The measured results demonstrate that in its $x(y)$-LP state, the peak realized gain, front-to-back ratio (FTBR), and radiation efficiency values are, respectively, $\sim 3.03 \mathrm{dBi}(2.97 \mathrm{dBi}), \sim 10.7 \mathrm{~dB}$ $(9.9 \mathrm{~dB})$, and $\sim 68.2 \%(67.5 \%)$. For the LHCP (RHCP) states they are, respectively, $\sim 2.82 \mathrm{dBi}(2.74 \mathrm{dBi}), \sim 11.4 \mathrm{~dB}(12.5 \mathrm{~dB})$, and $\sim 67.1 \%(65.9 \%)$.
\end{abstract}

Index Terms - Electrically small antennas, low-profile antennas, Huygens dipole antennas, near-field resonant parasitic elements, polarization-reconfigurable antennas.

Manuscript received on Feb. 12, 2018; revised on Jul. 24, 2018; and accpated on Sep. 4, 2018.

This work was supported in part by the National Natural Science Foundation of China, contract number 61471072; in part by Graduate Scientific Research and Innovation Foundation of Chongqing, China contract number CYB18069; in part by Opening subject of State Key Laboratory of Millimeter Waves, contract number K201732; in part by the Funding of the Innovative Leading Talents in Science and Technology of Chongqing, contract number CSTCCXLJRC201705; in part by the Funding of the leading research talent cultivation plan of Chongqing University contract number cqu2017hbrc1 A08; in part by Funding of the Young Backbone Teachers in Colleges and Universities of Chongqing, contract number 0307001104102; in part by the Fundamental Research Funds for the Central Universities contract number 2018CDQYTX0025; and in part by the Australian Research Council grant number DP160102219.

M. -C. Tang, Z. Wu, and T. Shi are with the Key Laboratory of Dependable Service Computing in Cyber Physical Society Ministry of Education, College of Communication Engineering, Chongqing University, Chongqing 400044, China and also with the State Key Laboratory of Millimeter Waves, Nanjing, 210096, China (E-mail: tangmingchun@cqu.edu.cn);

R. W. Ziolkowski is with the University of Technology Sydney, Global Big Data Technologies Centre, Ultimo NSW 2007, Australia, and the Department of Electrical and Computer Engineering, University of Arizona, Tucson, AZ 85721, USA (E-mail: Richard.Ziolkowski@uts. edu.au).

\section{INTRODUCTION}

$\mathrm{E}$ lectrically small antennas (ESAs) with high-directivity have received increasing attention in recent years. They provide significant advantages for many space-limited wireless platforms associated, for example, with long-distance and point-to-point communication systems [1,2]. The pursuit of higher directivity ESAs has led to a variety of designs. Nevertheless, Huygens sources, which utilize pairs of magnetic and electric radiators to achieve the desired directive outcome, possess intrinsic advantages. In particular, the high directivity is obtained without the need to load the radiator with additional constructs such as periodic electromagnetic band gap (EBG) structures [3], slot structures [4], or reflector elements [5]. These constructs generally increase the overall size and profile of the antenna system significantly. A variety of Huygens source ESAs have been reported with different arrangements, including spherical wires [6], curved wires [7], and planar strips [8]. We recently have also developed several Huygens dipole ESAs using near-field resonant parasitic (NFRP) technologies [9]. These include three-dimensional structures [10, 11], multi-layered structures [12, 13], and non-Foster designs [14]. However, it is expected that Huygens source ESAs could be even more useful if they were versatile. For instance, antennas with polarization reconfigurability have many advantages. These include mitigating polarization mismatch, improving system capacity, reducing channel interference, and realizing multiple transmission channels for frequency reuse [15]. Therefore, it would be highly desirable to enable polarization reconfigurability in an electrically small Huygens source antenna. Such a polarization diverse ESA would be quite suitable for many modern compact wireless applications [16, 17], especially for narrowband multiple-input multiple-output (MIMO) communication systems [18] and narrowband wireless fidelity (WiFi) connections [19].

A number of antennas with polarization reconfigurability have been reported in the literature over several decades [20-27]. Generally, the methods to realize polarization diversity can be divided into three categories. The first employs reconfigurable radiating elements [20-22]. The second relies on reconfigurable feeding networks [23-25]. The third resorts to reconfigurable parasitic periodic structures [26-27]. Unfortunately, none of these methods prove to be useful individually in the design of a polarization-reconfigurable Huygens source ESA. Specifically, the first method would 
necessitate the placement of p-i-n (PIN) diodes/switches on both the magnetic and the electric NFRP elements, the actual radiators. Thus, a large number of PIN diodes would be needed to simultaneously control both of them. In turn, this would lead to an increase in the difficulties in the design and assembly of the antenna, especially with the placement of the requisite bias lines. The second method requires components such as power dividers, phase delay lines, and other related devices to realize a reconfigurable feed network. They result in a significant increase in the electrical size and design complexity of the overall system. The third method integrates reconfigurable periodic elements, e.g., active EBG structures [26] or metasurfaces [27], into the antenna system. They make the total antenna size electrically large. Thus, to the best our knowledge, there has been no ESA design with polarization reconfigurability reported to date, let alone a reconfigurable Huygens source ESA.

A four-state, reconfigurable Huygens dipole antenna is reported in this paper. Different from the three methods discussed previously, a reconfigurable driven element is introduced that selectively excites the requisite NFRP elements to achieve the different polarization states. Benefiting from the inherent geometrical disconnect between the driven and NFRP elements, the antenna reconfigurability is then easily accomplished without any modifications to the NFRP elements. Six PIN diodes are introduced to change the current pathways on the driven element, which in turn lead to four states of polarization, i.e., two linear polarized (LP) states and two circular polarized (CP) states, by changing the currents on the NFRP elements through the different capacitive coupling behaviors between them and the driven element. The antenna configuration is described in Section II, and its design parameters are detailed. As discussed in Section III, a prototype was fabricated and tested. The measured results, in good agreement with their simulated values, confirm the efficacy of this electrically small, polarization-reconfigurable, Huygens dipole NFRP design with quad-polarization diversity. Finally, some conclusions are drawn in Section IV.

All of the numerical simulations and their optimizations reported herein were performed using the frequency domain, finite-element based ANSYS/ANSOFT High Frequency Structure Simulator (HFSS), version13.0. The simulation models employed the known, real properties of the dielectrics and conductors. An operational frequency near $1.5 \mathrm{GHz}$ was selected for the designs to simplify the fabrication of the antenna and to match the available measurement equipment.

\section{ReConfigurable Huygens SOURCE ESA DESIGN}

Fig. 1 illustrates the configuration of the polarization-reconfigurable Huygens dipole ESA. The corresponding optimized design parameters are given in Table I. As shown in Figs. 1(a) and 1(b), the antenna consists of three substrate layers which are labeled as Layer_1, Layer_2 and Layer_3, respectively. All of these substrates are Taconic TLY -5 with relative dielectric constant $\varepsilon_{r}=2.2$, loss tangent tan $\delta=0.0009$, and copper cladding thickness $0.017 \mathrm{~mm}$. All three layers have the same radius, $\mathrm{R} 1=30.0 \mathrm{~mm}$. However, each of them has a different thickness: $\mathrm{h} 1=\mathrm{h} 2=0.25 \mathrm{~mm}$, and $\mathrm{h} 3=$ $1.58 \mathrm{~mm}$.

The magnetic element consists of two intersecting, orthogonally-oriented capacitively loaded loops (CLLs) whose sizes are the same. One is oriented along the $x$-axis, and the other is along the $y$-axis. As shown in Fig. 1(c), the upper face of the magnetic element is cross-shaped with a copper ring (inner radius R4 and outer radius R3) in the center and is located on the upper surface of Layer_1. The four rectangular copper strips shown in Fig. 1(e) are located on the top of Layer_3 and act as the lower part of the magnetic element. Each are $\mathrm{L} 4 \times \mathrm{W} 1$ in size and are symmetrically located with respect to the center of the disk. The gaps between them along the $x$ and $y$-axis are equal (g2). The cross-shaped element on Layer_1 and these four copper strips on Layer_3 are connected by four copper cylindrical columns, each with height $\mathrm{h} 4=7.4 \mathrm{~mm}$ and radius $\mathrm{R} 2=1.25 \mathrm{~mm}$. These four copper cylindrical columns, respectively, pass completely through the four copper rings shown in Fig. 1(b) that are located on the top of Layer_2. The rings have an inner radius $\mathrm{R} 10=1.8 \mathrm{~mm}$ and outer radius $\mathrm{R} 9=$ $2.8 \mathrm{~mm}$. They are printed on the electric element to avoid the magnetic element being shorted to it.

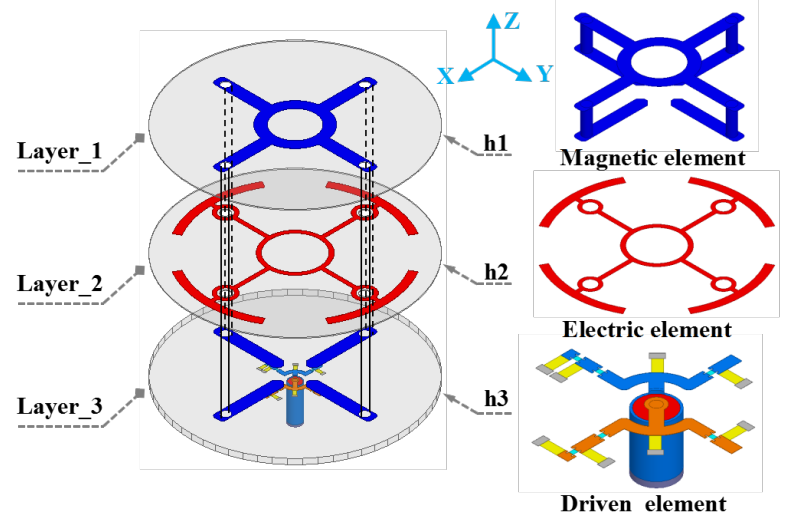

(a)

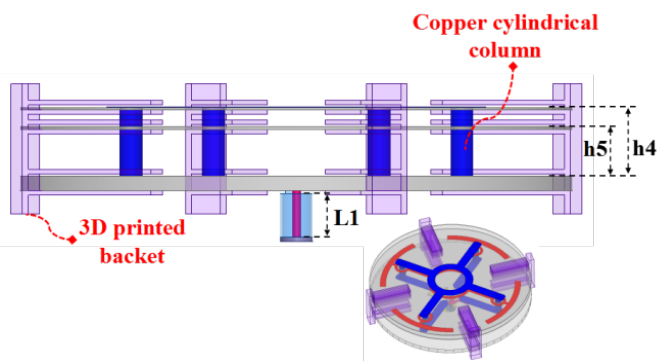

(b)

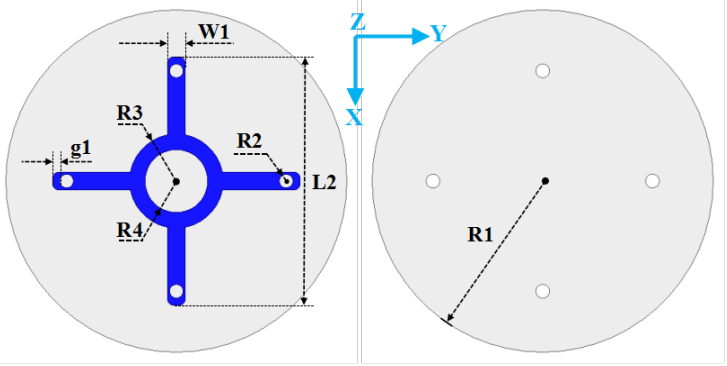

(c) 

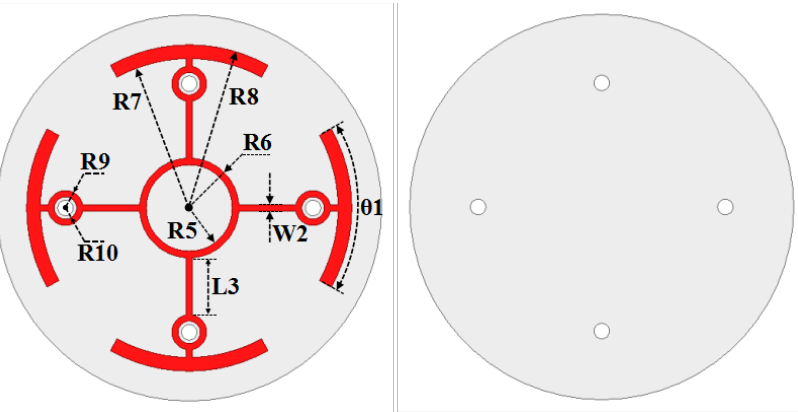

(d)
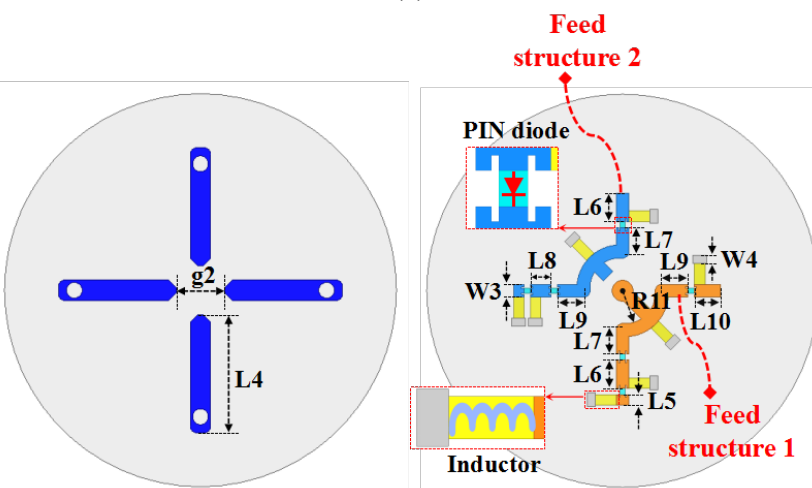

(e)

Fig.1 Geometry of the polarization-reconfigurable Huygens dipole ESA. (a) 3-D isometric view. (b) Side view. Upper and lower surfaces of (c) Layer_1, (d) Layer_2, and (e) Layer_3.

The electric element consists of two Egyptian axe dipoles (EADs) printed on the upper surface of Layer_2, each having the same size. One is oriented along the $x$-axis, and the other is along the $y$-axis. As shown in Fig. 1(d), it also has a copper ring (inner radius $\mathrm{R} 5$ and outer radius $\mathrm{R} 6$ ) in its center.

These dual CLLs and EADs operate as the NFRP elements. They are excited by the driven element, which is located on the lower surface of Layer_3. To ensure the mechanical stability of the antenna system during its assembly and testing, a KTG 141-50 type, semi-rigid, $50 \Omega$ coaxial cable was selected to feed it and four 3D printed polyamide brackets were used to maintain the relative vertical distance between the three layers. Because the operating mechanisms of the CLL and EAD NFRP elements together with their design guidelines when used to form a Huygens dipole ESA have been described previously [11], they will not be repeated here.

TABle I. The Optimized Design PARAmeters (IN Millimeters) OF THE POLARIZATION-RECONFIGURABLE HuYGENS Dipole ESA

\begin{tabular}{|c|c|c|c|c|}
\hline$h 1=0.25$ & $h 3=1.58$ & $h 4=7.4$ & $h 5=5.3$ & $L 1=5$ \\
\hline$L 2=45.2$ & $L 3=9.43$ & $L 4=19$ & $L 5=0.8$ & $L 6=2.16$ \\
\hline$L 7=1.8$ & $L 8=1.6$ & $L 9=2$ & $L 10=2$ & $W 1=3.2$ \\
\hline$W 2=1.07$ & $W 3=1$ & $W 4=0.6$ & $R 1=30$ & $R 2=1.25$ \\
\hline$R 3=8.5$ & $R 4=5.7$ & $R 5=6.93$ & $R 6=8$ & $R 7=24$ \\
\hline$R 8=26.1$ & $R 9=2.8$ & $R 10=1.8$ & $R 11=3.3$ & $g 1=1.27$ \\
\hline$g 2=7.35$ & $\theta 1=60^{\circ}$ & \multicolumn{3}{|c|}{ Null } \\
\hline
\end{tabular}

\section{A. Reconfigurable Driven Element Design}

In order to realize the polarization reconfigurability amongst the two LP and two CP modes, a reconfigurable driven element was developed. Its configuration is highlighted in orange (feed structure 1) and blue (feed structure 2) in Fig. 1(e). The 50- $\Omega$ coaxial feed cable is placed at the center of the lower surface of Layer_3 and is oriented orthogonal to it. Its inner and outer conductors are connected, respectively, to the orange and blue circular segments with inner radius $\mathrm{R} 11=3.3 \mathrm{~mm}$ that are concentric to the center of the layer. Two pairs of copper strips of length L7 and L9 extend from these circular segments along the $x$-axis and $y$-axis, respectively. Another two equal length (L6) copper strips are also oriented along the $\pm x$-axis. Similarly, another two copper strips, but with the different lengths, i.e. $\mathrm{L} 8 \neq \mathrm{L} 10$, are also oriented along the $\pm y$-axis. Two additional rectangular copper strips, each $\mathrm{L} 5 \times \mathrm{W} 3$ in size, are printed along the $-y$-axis and $+x$-axis, respectively. Furthermore, there are eight additional rectangular copper patches (gray) distributed on the lower surface of Layer_3. As shown in Fig. 1(e), the Coil Craft inductors are installed between these patches and the driven element strips to block any RF signal from entering into the dc bias network. Each patch is used as the connection point for the dc feeder lines required to control the states of the diodes.

Six M/A-COM MA4GP907 PIN diodes were incorporated into this driven element. As shown in the subplot of Fig. 1(e), two pairs of small rectangular slots were etched on both sides of the gaps in order to ensure their accurate positions when they were installed. All four slots were $0.1 \mathrm{~mm} \times 0.2 \mathrm{~mm}$ in size. Two pads, $0.385 \mathrm{~mm} \times 0.2 \mathrm{~mm}$ in size, were located on each side of these gaps. They acted as markers for the placement of each diode.

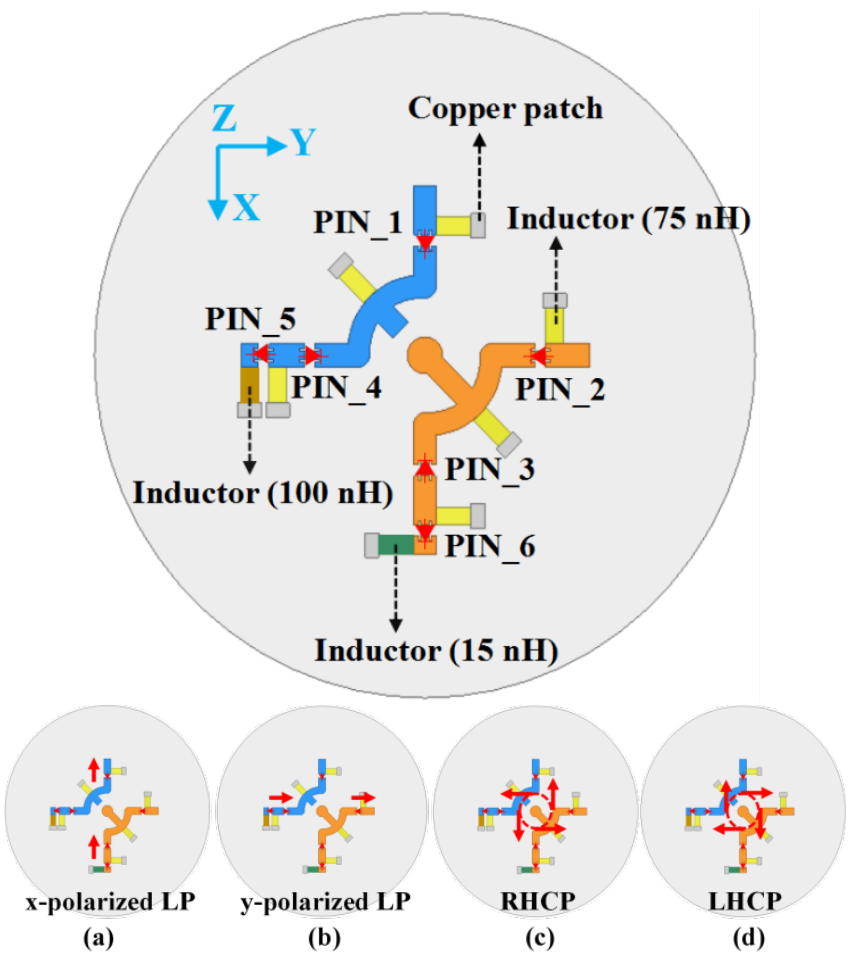

Fig. 2 The locations of the PIN diodes and the RF blocking inductors on the driven element are indicated in the top subplot. The current directions for each of the four polarization states are indicated by the red arrows in the bottom row of subplots. (a) $x$-polarized LP. (b) $y$-polarized LP. (c) RHCP. (d) LHCP. 
For an easy description of their ON and OFF states, we have denoted these diodes as PIN 1 - PIN 6 and show their location in Fig. 2. According to the PIN datasheet, each diode acts as a 4ohmresistor in its ON state and $0.025 \mathrm{pF}$ capacitor in its OFF state [28]. The two LP states are produced and switched by changing the states of the first four PINs (i.e., PIN_1 to PIN_4), as detailed in Figs. 2(a) and 2(b). When only PIN_1 and PIN_3 are turned $\mathrm{ON}$ and the rest are turned OFF, the antenna generates a Huygens radiation pattern whose maximum is directed along the $+\mathrm{z}$-axis and its LP state is oriented along the $x$-axis. Similarly, when only PIN_2 and PIN_4 are switched $\mathrm{ON}$ and the rest are turned OFF, the antenna generates a Huygens radiation pattern directed along the $+\mathrm{z}$-axis and its LP state is oriented along the $y$-axis.

The two CP states are produced by turning the first four PINs ON and changing the ON/OFF states of PIN_5 and PIN_6. These arrangements are depicted in Figs. 2(c) and 2(d). According to the design principles reported in [29], a CP ESA is achieved by feeding two orthogonally oriented LP radiators with a $\pi / 2$ phase shift. With the first four PINs turned on, two orthogonally oriented Huygens LP patterns are generated. The states of the remaining two diodes, PIN_5 and PIN_6, control the $\pi / 2$ phase shift and, hence, which of the two CP modes are produced. In particular, whenPIN_6 is switched ON and PIN_5 is switched OFF, a LHCP Huygens field is radiated with its maximum along the $+z$ direction. On the other hand, when PIN 5 is switched ON and PIN 6 is switched OFF, a RHCP Huygens field is radiated with its maximum also in the $+z$ direction. The control of the radiated polarization with the PIN diode states is summarized in Table II.

TABLE II The PIN Diode STATES to AtTAIN THE Four Polarization STATES

\begin{tabular}{|l|c|c|c|c|c|c|c|}
\hline Category & State & $P I N \_1$ & $P I N \_2$ & $P I N \_3$ & $P I N \_4$ & $P I N \_5$ & $P I N \_6$ \\
\hline Fig. 2(a) & $X-L P$ & $O N$ & $O F F$ & $O N$ & $O F F$ & $O F F$ & $O F F$ \\
\hline Fig. 2(b) & $Y-L P$ & $O F F$ & $O N$ & $O F F$ & $O N$ & $O F F$ & $O F F$ \\
\hline Fig. 2(c) & RHCP & $O N$ & $O N$ & $O N$ & $O N$ & $O N$ & $O F F$ \\
\hline Fig. 2(d) & LHCP & $O N$ & $O N$ & $O N$ & $O N$ & $O F F$ & $O N$ \\
\hline
\end{tabular}

\section{B. Polarization Reconfigurability Illustrated}

In order to clarify the operational mechanisms of the four polarization states, the current distributions on the antenna were simulated for each of them. Since both of the LP and CP states have similar operating principles, only the $y$-polarized LP and the RHCP states were selected for discussion herein.

The surface current distributions on the main copper surfaces were simulated and are depicted on the upper surface of the driven element and on the upper surfaces of the NFRP elements for the $y$-polarized Huygens LP and the RCHP states, respectively, in Figs. 3 and 4 at $1.503 \mathrm{GHz}\left(\lambda_{0}=199.60 \mathrm{~mm}\right)$, which is a frequency point within all four operational bands. To facilitate understanding, the current directions are highlighted with the additional solid red arrows in Fig. 3. The currents are found to be uniform, directed along they-axis, and maintained over the entire period. This means that the segments of the CLL and EAD elements located along the $y$-axis are excited by the $y$-oriented driven strips and generate the y-polarized Huygens LP radiation pattern. BecausePIN_1 and PIN_3 are turned
OFF, the driven element segments along the $x$-axis and, hence, the corresponding pieces of the CLL and EAD elements are not excited.

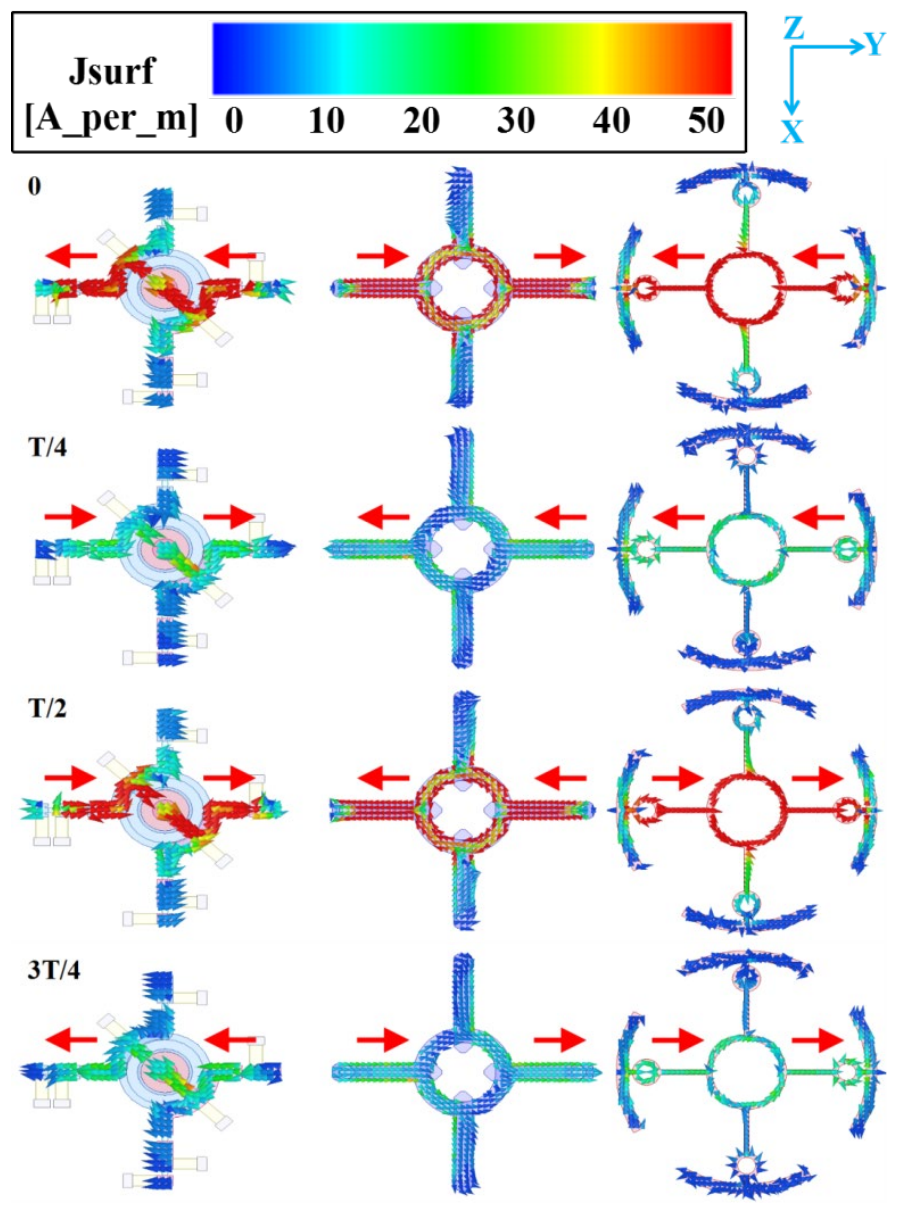

Fig. 3 Surface current distributions on the driven element and on the upper faces of CLL and EAD NFRP elements at quarter period intervals when the reconfigurable Huygens dipole ESA is in its $y$-polarized LP state.

Likewise, the current distributions on the driven strips and corresponding segments of the CLL and EAD elements are shown in Fig. 4 for the RHCP state. The more complicated surface current directions are highlighted with the solid red (referring to the driven element), purple (referring to the upper face of the CLL element), and orange (referring to the upper face of the EAD element) arrows to annotate their directional behavior. Recall that the first four PINs are switched ON to excite both the CLL and EAD elements to generate two orthogonal LP radiators with equal amplitude. The additional rectangular copper strip along the $-y$-axis that is connected when PIN 5 is turned ON produces the requisite $\pi / 2$ phase difference to attain the RHCP state. In detail, when PIN_5 is turned ON and PIN_6 is OFF, the lengths of the copper strips along the $y$-axis are longer than those along the $x$-axis. As a consequence, the long $y$-axis branches operate on the lower frequency side of the resonance frequency (i.e., they act capacitive components) while the short branches along the $x$-axis operate on the higher frequency side (i.e., they act as inductive components). Their sizes were optimized to achieve 
the requisite $\pi / 2$ phase shift at the frequency $1.503 \mathrm{GHz}$. Moreover, following the purple arrows as an example, their vector directions along the $y$-axis and $x$-axis rotate in the counterclockwise direction. Hence, the RHCP field is generated. As the system is a broadside radiator, the maximum directivity is along the $+z$ direction. In the same manner, when PIN_6 is turned ON and PIN_5 is OFF, the $x$-axis branches become longer than the $y$-axis branches, leading to the generation of the LHCP field.

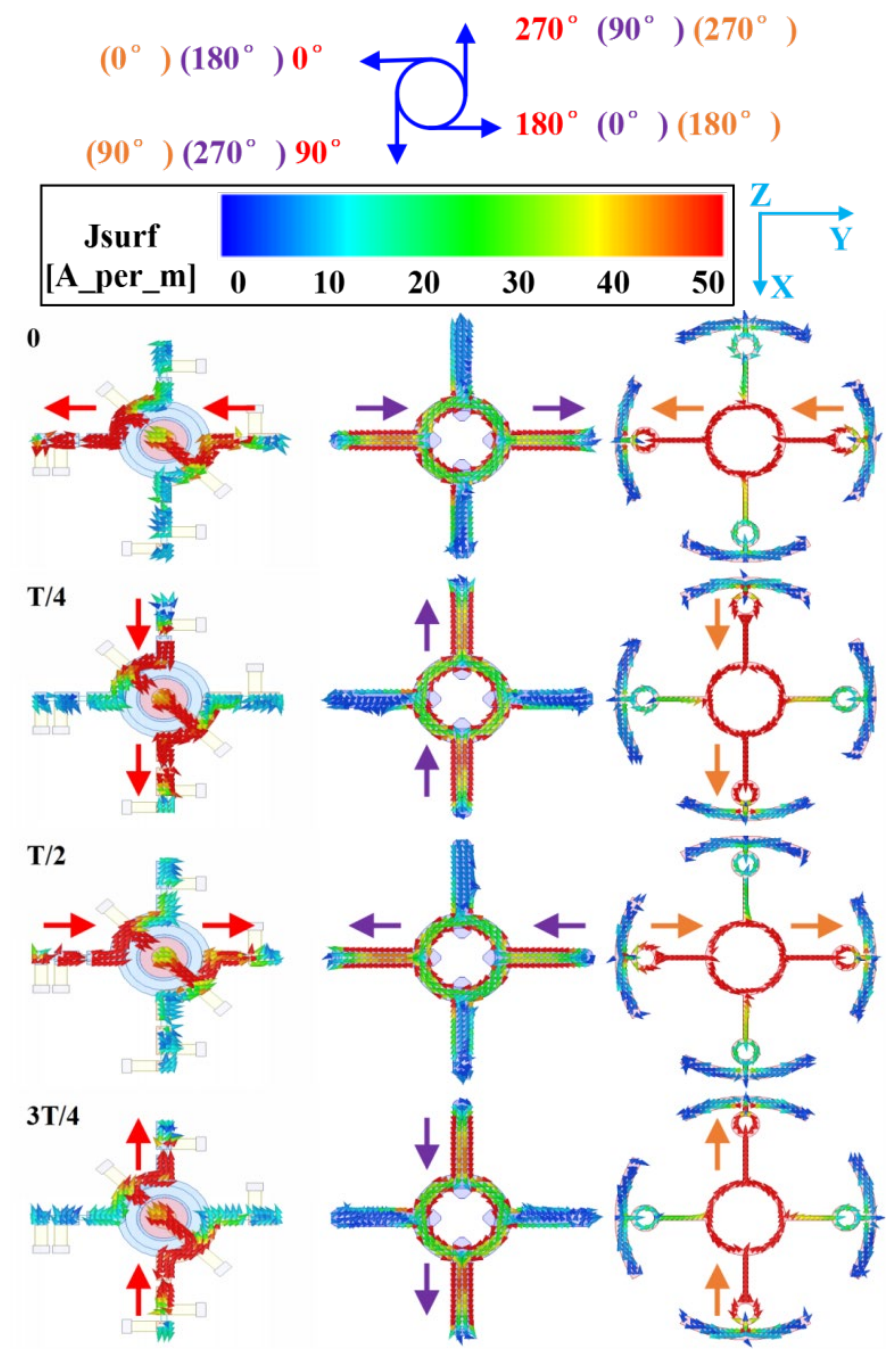

Fig. 4 Surface current distributions on the driven element and on the upper faces of CLL and EAD NFRP elements at quarter period intervals when the reconfigurable Huygens dipole ESA is in its RHCP state.

\section{SimULATED AND MEASURED RESULTS OF THE \\ POLARIZATION-RECONFIGURABLE HUYGENS DiPOLE ESA}

The optimized polarization-reconfigurable Huygens dipole ESA was fabricated, assembled, and measured. The assembled antenna system is shown in Figs. 5(a) and 5(b). The antenna under test (AUT) in the measurement chamber is shown in Fig. 5(c) along with the ATTEN TPR3003T-3C regulated dc power supply that provided the requisite $\sim 1.4 \mathrm{~V}$ to turn on the PIN diodes. The $\left|\mathrm{S}_{11}\right|$ values (reflection coefficients) of the antenna in its different polarization states as functions of the source frequency were measured with an Agilent E8361A PNA Vector
Network Analyzer (VNA). The far-field radiation performance of the antenna was measured with a SG128 multi-probe antenna measurement system at the China Academy of Information and Communications Technology, Chongqing, China [31]. This antenna measurement system can directly measure the realized gain, directivity, axial ratio (AR), antenna radiation efficiency (RE), and other performance characteristics.

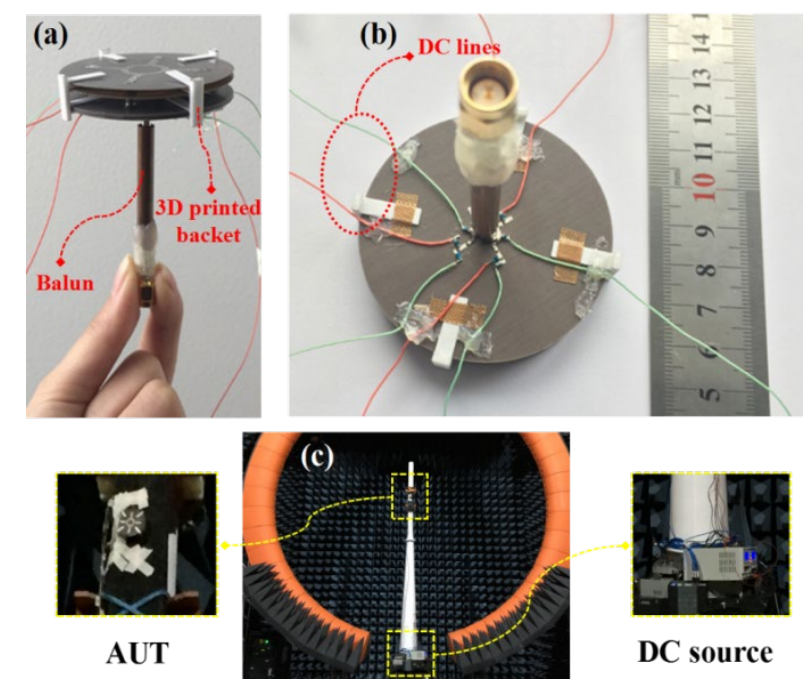

Fig. 5 Fabricated prototype of the polarization-reconfigurable Huygens dipole ESA. (a) The side view of the assembled antenna. (b) The bottom view of the assembled antenna. (c) The antenna under test (AUT) in the chamber.

The presence of the balun shown in Fig. 5 was necessary only to evaluate the antenna performance accurately. The long coaxial cable associated with the measurement system would have led to inescapable leakage currents on it that would have influenced the radiation characteristics for such a balanced antenna structure [11]. Therefore, a sleeve balun that was 49 $\mathrm{mm}$ in length (nearly one-quarter wavelength) was designed to mitigate any of those spurious currents induced on the outer walls of that long coax cable. Consequently, the accuracy of the measured results was improved significantly [30].Furthermore, because the balun was only ancillary to those measurements, it was not included in the calculation of its $k a$ value.

The simulated (taking into account of the presence of the balun) and the measured $\left|S_{11}\right|$ values for the two LP and the two CP polarization states are presented in Figs. 6(a) and 6(b), respectively. The simulated (measured)resonance frequencies of the $x$ - and $y$ - LP states are $1.508 \mathrm{GHz}(1.507 \mathrm{GHz})$, and 1.509 $\mathrm{GHz}(1.512 \mathrm{GHz})$, respectively, where $\left|\mathrm{S}_{11}\right|_{\min }=-41.6 \mathrm{~dB}(-12.4$ $\mathrm{dB})$, and $-30.0 \mathrm{~dB}(-12.0 \mathrm{~dB})$. The measured $-10 \mathrm{~dB}$ bandwidths are $1.502-1.516 \mathrm{GHz}(14 \mathrm{MHz}, 0.92 \%)$ and $1.503-1.520 \mathrm{GHz}$ (17 MHz, $1.1 \%)$ for these $x$ - and $y$ - LP states, respectively. Thus, the measured overlapped operating bandwidth for the two LP states is $13 \mathrm{MHz}$, covering $1.503-1.516 \mathrm{GHz}$. Similarly, the simulated (measured) resonance frequencies of the LHCP and RHCP states are $1.501 \mathrm{GHz}(1.501 \mathrm{GHz})$, and $1.501 \mathrm{GHz}$ $(1.500 \mathrm{GHz})$, where $\left|\mathrm{S}_{11}\right|_{\min }=-19.5 \mathrm{~dB}(-16.5 \mathrm{~dB})$, and $-16.5 \mathrm{~dB}$ $(-13 \mathrm{~dB})$. The measured $-10 \mathrm{~dB}$ bandwidths are $1.491-1.511$ $\mathrm{GHz}(20 \mathrm{MHz}, 1.33 \%)$ and $1.493-1.509 \mathrm{GHz}(16 \mathrm{MHz}, 1.06 \%)$ for these LHCP and RHCP states, respectively. Thus, the 
measured overlapped operating bandwidth for the LHCP and RHCP states is $16 \mathrm{MHz}$, covering $1.493-1.509 \mathrm{GHz}$.

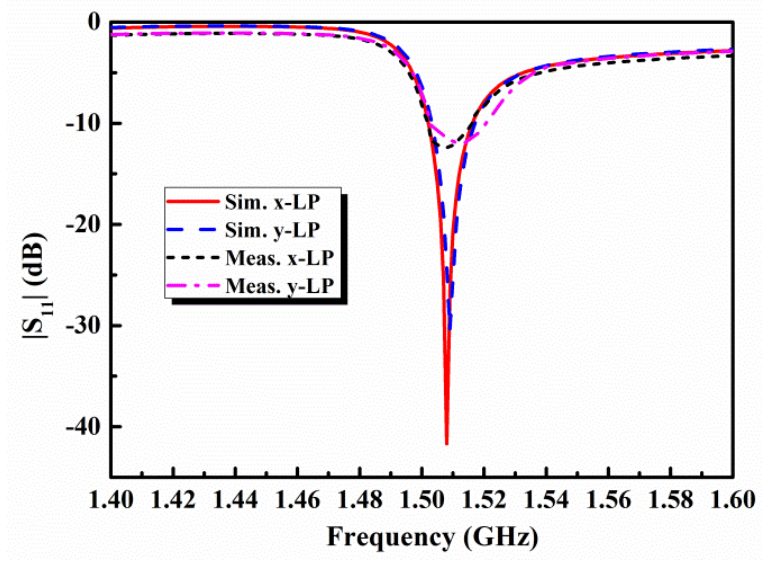

(a)

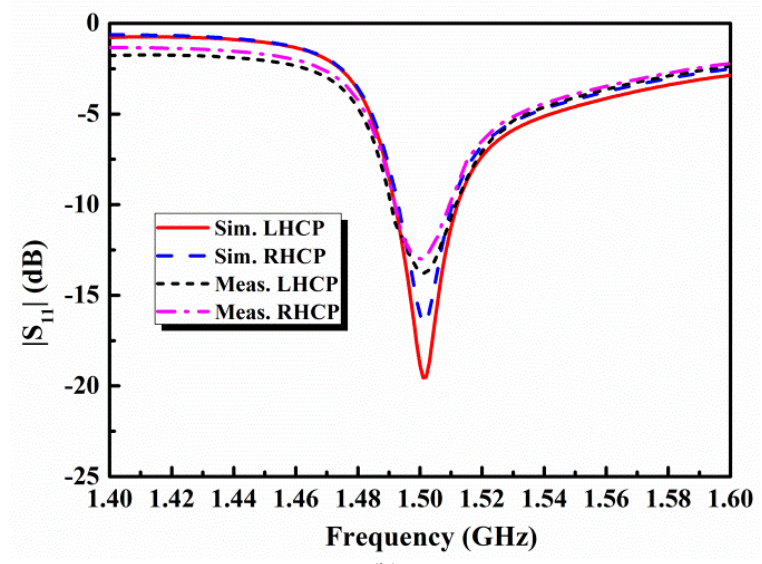

(b)

Fig. 6 Simulated and measured $\left|S_{11}\right|$ values of the polarization-reconfigurable Huygens source ESA for its four polarization states as functions of the source frequency. (a) $x$ - and $y$ - LP states. (b) LHCP and RHCP states.

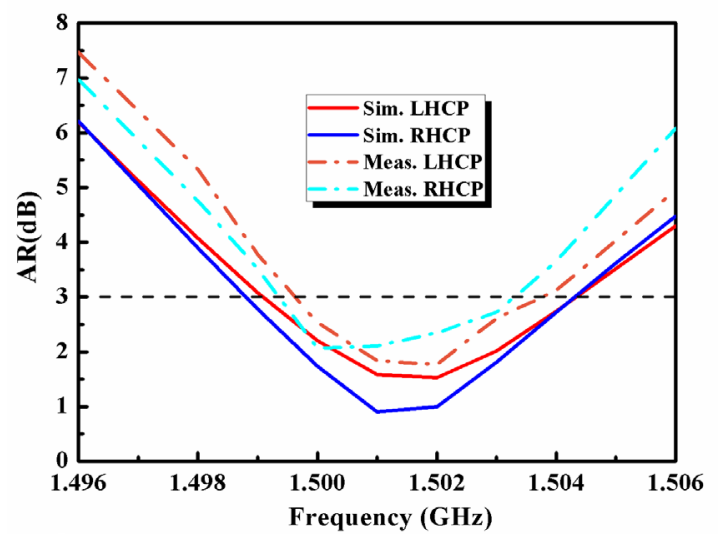

Fig. 7 Simulated and measured AR values when the polarization-reconfigurable Huygens source ESA is radiating its LHCP and RHCP states.

The AR values of the two $\mathrm{CP}$ states are plotted in Fig. 7 as functions of the source frequency. The simulated 3-dB AR bandwidths are $1.4991-1.5043 \mathrm{GHz}(5.2 \mathrm{MHz}, 0.34 \%)$ and $1.4988-1.5043 \mathrm{GHz}(5.5 \mathrm{MHz}, 0.36 \%)$ for the LHCP and RHCP states, respectively. The simulated overlapped operating 3-dB AR bandwidth is $1.4991-1.5043 \mathrm{GHz}(5.2 \mathrm{MHz}, 0.34 \%)$.
The measured overlapped operating 3-dB AR bandwidth is $1.500-1.503 \mathrm{GHz}(3.0 \mathrm{MHz}, 0.19 \%)$.

Fig. 8 presents the simulated and measured realized gain patterns when the antenna radiates each of its four polarization states. The main-beam direction for each of these states is clearly oriented along the $+z$-axis. Good agreement between the simulated and measured results is demonstrated.

The measured realized gain patterns of the $x$ - and $y$-LP states are shown in Figs. 8(a) and 8(b), respectively. For the $x$-LP state, the simulated (measured) peak realized gain, FTBR, and RE values are $\sim 3.51 \mathrm{dBi}(3.03 \mathrm{dBi}), 11.2 \mathrm{~dB}(10.7 \mathrm{~dB})$, and $\sim 77.9 \%(68.2 \%)$, respectively. The corresponding values for the $y$-LP state are $\sim 3.54 \mathrm{dBi}(2.97 \mathrm{dBi}), \sim 11 \mathrm{~dB}(9.9 \mathrm{~dB})$, and $\sim 78.9 \%(67.5 \%)$, respectively. In the $x$-LP state, the simulated (measured) half-power beamwidth was from $-61.5^{\circ}$ to $62.9^{\circ}$ (from $-79^{\circ}$ to $50^{\circ}$ ) in the E-plane (z0x-plane) and from $-76.6^{\circ}$ to $76.5^{\circ}$ (from $-51^{\circ}$ to $71^{\circ}$ ) in the H-plane ( $z 0 y$-plane). In the $y$-LP mode, the simulated (measured) half-power beamwidth was from $-60.5^{\circ}$ to $62.3^{\circ}$ (from $-60^{\circ}$ to $50^{\circ}$ ) in the E-plane (z0y-plane) and from $-77.4^{\circ}$ to $77.7^{\circ}$ (from $-52^{\circ}$ to $80^{\circ}$ ) in the $\mathrm{H}$-plane ( $z O x$-plane). The cross-polarization levels in the broadside direction are below $-15 \mathrm{~dB}$ for both LP states, indicating their high polarization purity.

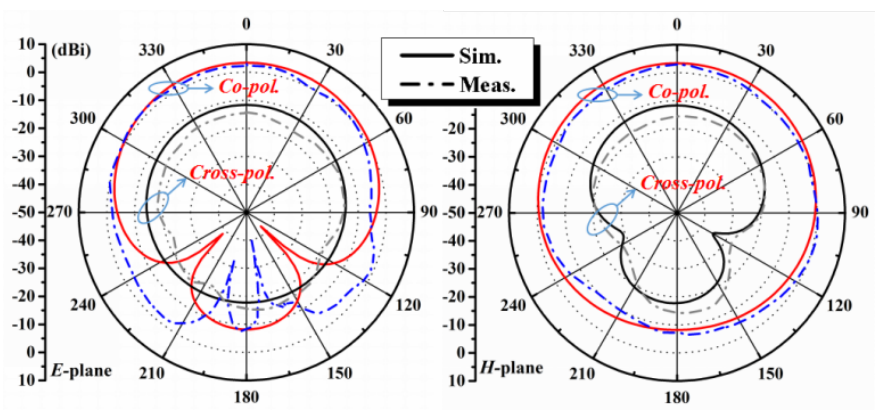

(a)

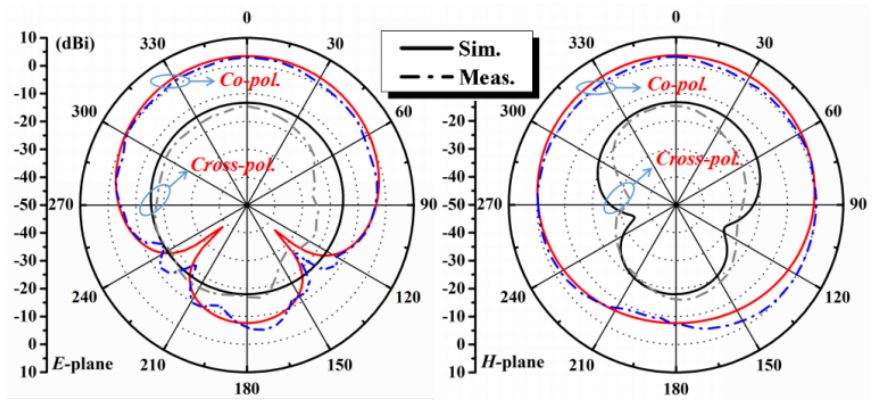

(b)

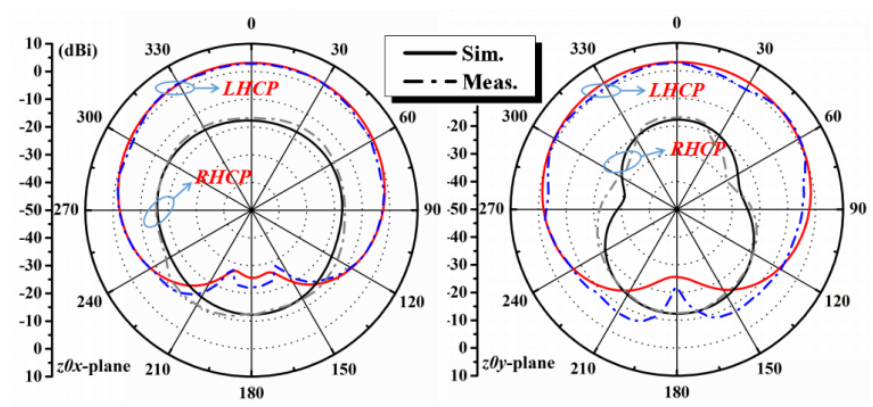

(c) 


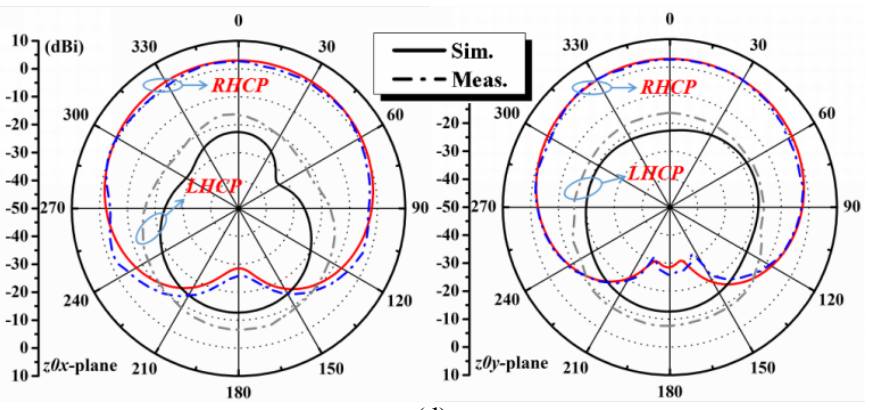

(d)

Fig. 8 Simulated and measured realized gain patterns for the four polarization states at specific measured (simulated) frequencies in their overlapping frequency bands.(a) $x$-LP at 1.507 (1.508) GHz. (b) $y$-LP at 1.512 (1.509) GHz. (c) LHCP at 1.501 (1.501). (d) RHCP at 1.500 (1.501) GHz.

The measured realized gain patterns of the LHCP and RHCP states are shown in Figs. 8(c) and 8(d), respectively. For the LHCP state, the simulated (measured) peak realized gain, FTBR, and RE values are $\sim 3.13 \mathrm{dBi}(2.82 \mathrm{dBi}), \sim 15.2 \mathrm{~dB}(11.4$ $\mathrm{dB})$, and $\sim 71.3 \%(67.1 \%)$, respectively. The corresponding values for the RHCP state are $\sim 2.99 \mathrm{dBi}(2.74 \mathrm{dBi}), \sim 15.5 \mathrm{~dB}$ $(12.5 \mathrm{~dB})$, and $\sim 69.8 \%(65.9 \%)$, respectively. In the LHCP state, the simulated (measured) half-power beamwidth was from $-68.1^{\circ}$ to $68.3^{\circ}$ (from $-57^{\circ}$ to $61^{\circ}$ ) in the $z 0 x$-plane and from $-71.1^{\circ}$ to $68.7^{\circ}$ (from $-68^{\circ}$ to $68^{\circ}$ ) in the $z 0 y$-plane. In the RHCP state, the simulated (measured) half-power beamwidth was from $-68.6^{\circ}$ to $70.6^{\circ}$ (from $-72^{\circ}$ to $61^{\circ}$ ) in the $z 0 x$-plane and from $-68.4^{\circ}$ to $68.6^{\circ}$ (from $-57^{\circ}$ to $56^{\circ}$ ) in the $z 0 y$-plane.

The simulated and measured RE values were stable and high ( $>70 \%$ in simulation and $>60 \%$ in measurement) in all of its dynamic polarization states. As illustrated in Figs. 3 and 4, the electrical and magnetic NFRP elements play the main role for radiating the energy outwards in the LP and CP states. As explained previously [9], this NFRP feature is responsible for the RE being much larger than it would be if only a driven structure was employed. This aspect was demonstrated in a previous reconfigurable ESA design [32]. It facilitates two significant advantages of this design. The first is that since all the lumped elements are integrated on the driven structure and not on the NFRP elements, they cause much less energy loss. The second is actually related the first one. Because the six PIN diodes are integrated into the driven elements, there is only a small difference in the RE values between the $\mathrm{ON}$ and $\mathrm{OFF}$ states. They have no impact on the NFRP elements.

To emphasize the efficacy of our electrically small $(k a=$ 0.944) system, we note that two electrically large magneto-electric dipole antennas with polarization-diversity have been reported recently $[33,34]$. The volume of two antennas are, respectively, $1.89 \times 10^{-1} \lambda_{0}{ }^{3}(k a=4.081)$ [33] and $1.74 \times 10^{-1} \lambda_{0}{ }^{3}(k a=4.119)$ [34], which have the peak realized gain with the approximately same values $(\sim 8.2 \mathrm{dBi})$. The volume of our antenna, $4.05 \times 10^{-3} \lambda_{0}{ }^{3}$, is 46.6 times smaller than that in [33] and 42.96 times smaller than that in [34]. While our antenna's radiation efficiency is approximately the same to those in $[33,34]$, its aperture efficiency is much higher. In particular, while the physical aperture area of our antenna, $7.096 \times 10^{-2} \lambda_{0}{ }^{2}$, is 10.6 times smaller than that in [33] and 11.6 times smaller than that in [34], its realized gain is only $\sim 3.2$ times smaller than those in $[33,34]$.Moreover, our antenna does not require any cavity backing. Furthermore, our design is simpler, achieving the quad-diversity with only 6 , instead of 8PIN diodes in [33]. Even though a smaller number (4) of PIN diodes are utilized in [34], only tri-diversity was obtained. We note that while concurrently it has the expected smaller bandwidth because of its electrically smaller size, the compactness and multifunctional features of our design are quite attractive for the many existing and aspirational narrowband applications. On the other hand, by integrating non-Foster components into both the electric and magnetic NFRP elements [14], the operational bandwidths of the reported polarization-reconfigurable Huygens dipole ESA in all four of its dynamic states could be increased significantly. This design approach would facilitate a number of additional applications.

\section{CONCLUSION}

A polarization-reconfigurable Huygens dipole ESA was presented. Rather than altering previously successful Huygens dipole NFRP elements, the reported multi-functional configuration was achieved through the development of a novel, reconfigurable driven element implemented with six $\mathrm{PIN}$ diodes. By manipulating their ON/OFF states, it was demonstrated that this ESA is capable of dynamically achieving four different polarization states, i.e., two LP and two $\mathrm{CP}$ states, which are radiated with their maximum gain in the broadside direction. The measured overlapping impedance bandwidth for the two LP states is $0.86 \%$, covering 1.503-1.516 GHz; and for the two CP states is $1.06 \%$, covering 1.493-1.509 GHz. The measured, overlapping 3-dB AR bandwidth is about $0.19 \%$, from 1.500 to $1.503 \mathrm{GHz}$. The advantages of this low-profile, electrically small configuration include stable and useful realized gain values in its broadside direction, symmetric radiation patterns, and low back radiation in all four of its dynamically attainable polarization states. A prototype was fabricated and tested. The measured results are in good agreement with their simulated values, confirming the design strategy and the final optimized design. The multi-functionality attained with this polarization-reconfigurable approach could be extended to many other types of reconfigurable antennas. The realized system has a number of potential applications to the next generation wireless systems, e.g., to narrowband MIMO communication systems and narrowband WiFi connections.

\section{REFERENCES}

[1] Y. Yamada, W. G. Hong, W. H. Jung, and N. Michishita, "High gain design of a very small normal mode helical antenna for RFID tags," in Proc. IEEE TENCON, Taipei, Taiwan, Oct./Nov. 2007, pp. 1-4.

[2] W. Hong, Y. Yamada, and N. Michishita, "Low profile small normal mode helical antenna achieving long communication distance," in Proc. Int. Workshop Antenna Technol. (iWAT 2008), Chiba, Japan, Mar. 2008, pp. $167-170$.

[3] J. Ng and R. W. Ziolkowski, "Combining metamaterial-inspired electrically small antennas with electromagnetic band gap (EBG) structures to achieve higher directivities and bandwidths," in Proc. 2012 International Workshop on Antenna Technology (iWAT 2012), Tucson, 
America, pp. 189-192, Mar. 2012.

[4] M.-C. Tang and R. W. Ziolkowski, "Efficient, high directivity, large front-to-back-ratio, electrically small, near-field-resonant-parasitic antenna," IEEE Access, vol. 1, no. 1, pp. 16 - 28, May 2013.

[5] S. Lim and H. Ling, "Design of electrically small Yagi antenna," Electron. Lett., vol. 43, no. 5, pp. 3-4, Mar. 2007.

[6] S. R. Best, "Progress in the design and realization of an electrically small Huygens source" in Proc. 2010 International Workshop on Antenna Technology (iWAT 2010), Lisbon, Portugal, pp. 1-4, Mar. 1-3, 2010.

[7] P. Alitalo, A. O. Karilainen, T. Niemi, C. R. Simovski, and S. A. Tretyakov, "Design and realisation of an electrically small Huygens source for circular polarisation," IET Microw. Antennas Propag., vol. 5, no. 7, pp. 783-789, 2010

[8] T. Niemi, P. Alitalo, A. O. Karilainen, and S. A. Tretyakov, "Electrically small Huygens source antenna for linear polarisation," IET Microw. Antennas Propag., vol. 6, no. 7, pp. 735-739, 2012.

[9] R. W. Ziolkowski, P. Jin, and C.-C. Lin, "Metamaterial-inspired engineering of antennas," Proc. IEEE, vol. 99, pp. 1720-1731, Oct. 2011.

[10] R. W. Ziolkowski, "Low profile, broadside radiating, electrically small Huygens source antennas," IEEE Access, vol. 3, pp. 2644-2651, 2015.

[11] M.-C. Tang, H. Wang, and R. W. Ziolkowski, "Design and testing of simple, electrically small, low-profile, Huygens source antennas with broadside radiation performance," IEEE Trans. Antennas Propag., vol.64, no. 11, pp. 4607-4617, Nov. 2016.

[12] M.-C. Tang, T. Shi, and R. W. Ziolkowski, "A study of $28 \mathrm{GHz}$, planar, multi-layered, electrically small, broadside radiating, Huygens source antennas," IEEE Trans. Antennas Propag., in the Special Issue on "Antennas and Propagation Aspects of 5G Communications", vol. 65, no. 12, pp. 6345-6354, Dec. 2017.

[13] M.-C. Tang, B. Zhou, and R. W. Ziolkowski, "Low-profile, electrically small, Huygens source antenna with pattern-reconfigurability that covers the entire azimuthal plane," IEEE Trans. Antennas Propag., vol.65, no. 3, pp. 1063-1072, Mar. 2017.

[14] M.-C. Tang, T. Shi, and R. W. Ziolkowski, "Electrically small, broadside radiating Huygens source antenna augmented with internal non-Foster elements to increase its bandwidth," IEEE Antennas Wirel. Propag. Lett., vol.16, pp. 712-715, 2017.

[15] F. Wu and K. M. Luk, "A reconfigurable magneto-electric dipole antenna using bent cross-dipole feed for polarization diversity," IEEE Antennas Wireless Propag. Lett., vol. 16, pp. 412-415, 2017.

[16] C. G. Christodoulou, Y. Tawk, S. A. Lane, and S. R. Erwin, "Reconfigurable antennas for wireless and space applications," Proc. IEEE, vol. 100, no. 7, pp. 2250-2261, Jul. 2012.

[17] H. Wong, W. Lin, X. Wang, M. Lu, "LP and CP polarization reconfigurable antennas for modern wireless applications," in Proc. 2017 International Symposium on Antenna and Propagation (ISAP 2017), Phuket, Thailand, Oct. 30-Nov. 2 2017, pp. 1-2.

[18] A. Grau, J. Romeu, M.-J. Lee, S. Blanch, L. Jofre, and F. De Flaviis, “A dual-linearly-polarized MEMS-reconfigurable antenna for narrowband MIMO communication systems," IEEE Trans. Antennas Propag., vol. 58, no. 1, pp. 4-17, Jan. 2010

[19] Z. N. Chen, X. M. Qing, T. S. P. See, and W. K. Toh, "Antennas for WiFi connectivity," Proc. IEEE, vol. 100, no. 7, pp. 2322-2329, Jul. 2012.

[20] Y.-M. Cai, S. Gao, Y. Yin, W. Li, and Q. Luo, "Compact-size low-profile wideband circularly polarized omnidirectional patch antenna with reconfigurable polarizations," IEEE Trans. Antennas Propag., vol.64, no. 5, pp. 2016-2021, May 2016

[21] F. Wu and K. M. Luk, "Wideband tri-polarization reconfigurable magneto-electric dipole antenna," IEEE Trans. Antennas Propag., vol.65, no. 4, pp. 1633-1641, Apr. 2017.

[22] W. Lin and H. Wong, "Polarization reconfigurable wheel-shaped antenna with conical-beam radiation pattern," IEEE Trans. Antennas Propag., vol.63, no. 2, pp. 491-499, Feb. 2017.

[23] L.-Y. Ji, P.-Y. Qin, Y. J. Guo, C. Ding, G. Fu, and S.-X. Gong, "A wideband polarization reconfigurable antenna with partially reflective surface," IEEE Trans. Antennas Propag., vol. 64, no. 10, pp. 4534-4538, Oct. 2016.

[24] W. Yang, W. Che, H. Jin, W. Feng, and Q. Xue, "A polarization reconfigurable dipole antenna using polarization rotation AMC structure," IEEE Trans. Antennas Propag., vol. 63, no. 12, pp. 5305-5315, Dec. 2015.

[25] K. M. J. Ho and G. M. Rebeiz, "A 0.9-1.5 GHz microstrip antenna with full polarization diversity and frequency agility," IEEE Trans. Antennas Propag., vol. 62, no. 5, pp. 2398-2406, May 2014.

[26] B. Liang, B. Sanz-Izquierdo, E. A. Parker, and J. C. Batchelor, "A frequency and polarization reconfigurable circularly polarized antenna using active EBG structure for satellite navigation," IEEE Trans. Antennas Propag., vol. 63, no. 1, pp. 33-40, Jan. 2015.

[27] H. L. Zhu, S. W. Cheung, X. H. Liu, and T. I. Yuk, "Design of polarization reconfigurable antenna using metasurface," IEEE Trans. Antennas Propag., vol. 62, no. 6, pp. 2891-2898, Jun. 2014.

[28] MACOM. (2016). Products: MA4GP907. [Online]. Available: http://cdn.macom.com/datasheets/MA4GP907.pdf.

[29] P. Jin and R. W. Ziolkowski, "Multi-frequency, linear and circular polarized, metamaterial-inspired, near-field resonant parasitic antennas," IEEE Trans. Antennas Propag., vol. 59, no. 5, pp. 1446-1459, May 2011.

[30] C. Icheln, J. Krogerus, and P.Vainikainen, "Use of balun chokes in small-antenna radiation measurements," IEEE Trans. Antennas Propag., vol.53, no. 2, pp. 498-506, Apr. 2004.

[31] SG128 multi-probe antenna measurement system. [Online]. Available: https://www.mvg-world.com/en/system/files/datasheet_sg128_bd.pdf.

[32] M. -C. Tang, R. W. Ziolkowski, S. Xiao, M. Li, and J. Zhang, "Frequency-agile, efficient, near-field resonant parasitic monopole antenna," IEEE Trans. Antennas Propag., vol. 62, no. 3, pp. 1479 - 1483, Mar. 2014.

[33] F. Wu and K. M. Luk, "Single-port reconfigurable magneto-electric dipole antenna with quad-polarization diversity," IEEE Trans. Antennas Propag., vol. 65, no. 5, pp. 2289-2296, May 2017.

[34] Y. Shi, Y. Cai, X.-F. Zhang and K. Kang, "A simple tri-polarization reconfigurable magneto-electric dipole antenna," IEEE Antennas Wirel. Propag. Lett., vol.17, pp. 291-294, 2018. 


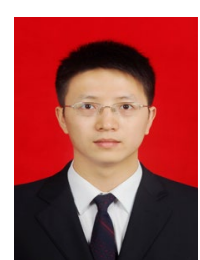

Ming-Chun Tang (S'12-M'13-SM'16) received the B. S. degree in physics from the Neijiang Normal University, Neijiang, China, in 2005 and the $\mathrm{Ph}$. D. degree in radio physics from the University of Electronic Science and Technology of China (UESTC), in 2013. From August 2011 to August 2012, he was also with the Department of Electrical and Computer Engineering, The University of Arizona, Tucson, AZ, USA, as a Visiting Scholar. He is currently a Professor in the College of Communication Engineering, Chongqing University, China. His research interests include electrically small antennas, RF circuits, metamaterial designs and their applications.

Prof. Tang was a recipient of the Best Student Paper Award in the 2010 International Symposium on Signals, Systems and Electronics (ISSSE2010) held in Nanjing, China. His Ph.D. student received Best Student Paper Award from the IEEE $7^{\text {th }}$ Asia-Pacific Conference on Antennas and Propagation (2018 IEEE APCAP) held in Auckland, New Zealand. He is the founding Chair of the IEEE AP-S / MTT-S Joint Chongqing Chapter. He serves on the Editorial Boards of several journals, including IEEE Access and IET Electronics Letters. $\mathrm{He}$ has also served on the review boards of many journals, including the IEEE Transactions on Antennas and Propagation, IEEE Transactions on Microwave Theory and Techniques, IEEE Antennas and Wireless Propagation Letters, IEEE Antennas and Propagation Magazine, IEEE Microwave and Wireless Components Letters, and many international conferences as a General Chair, TPC Member, Session Organizer, and the Session Chair.

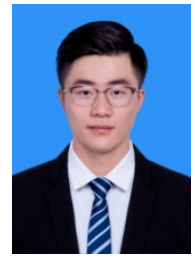

Zhentian Wu was born in China, in 1994. He received the B.S. degree from the West Anhui University, Liuan, China, in 2016. He is currently pursuing the M.S. degree in electronics and communication engineering in the College of Communication Engineering, Chongqing University, China.

He current research interests include electrically small antenna and directional antenna and their applications.

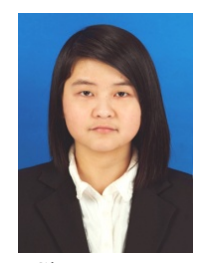

Ting Shi (S'16) received the B.S. degree from the University of Electronic Science and Technology of China (UESTC), Chengdu, China, in 2014 and the M.S. degree from the Chongqing University, Chongqing, China, in 2017. She is currently pursuing the $\mathrm{Ph}$. D degree in electronics and communication engineering in the College of Communication Engineering, Chongqing University, China.

She was a recipient of the Best Student Paper Award from the IEEE $7^{\text {th }}$ Asia-Pacific Conference on Antennas and Propagation (2018 IEEE APCAP) held in Auckland, New Zealand. Her current research interests include non-Foster circuits in electrically small antennas, active antenna applications.

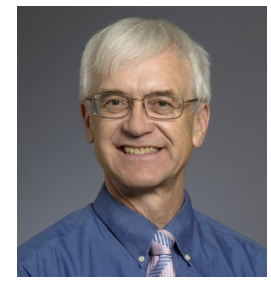

Richard W. Ziolkowski (M'87-SM'91-F'94) received the Sc. B. (magna cum laude) degree (Hons.) in physics from Brown University, Providence, RI, USA, in 1974; the M.S. and Ph.D. degrees in physics from the University of Illinois at Urbana-Champaign, Urbana, IL, USA, in 1975 and 1980, respectively; and an Honorary Doctorate degree from the Technical University of Denmark, Kongens Lyngby, Denmark in 2012.

Prof. Ziolkowski is the recipient of the 2019 IEEE Electromagnetics Award (IEEE Field Award). He is a Fellow of the Optical Society of America (OSA, 2006) and the American Physical Society (APS, 2016). He served as the President of the IEEE Antennas and Propagation Society in 2005. He is also actively involved with the URSI, OSA and SPIE professional societies. He was the Australian DSTO Fulbright Distinguished Chair in Advanced Science and Technology from 2014-2015. He was a 2014 Thomas-Reuters Highly Cited Researcher.

He is a Distinguished Professor in the Global Big Data Technologies Centre in the Faculty of Engineering and Information Technologies (FEIT) at the University of Technology Sydney, Ultimo NSW, Australia. He became a Professor Emeritus at the University of Arizona in 2018, where he was a Litton Industries John M. Leonis Distinguished Professor in the Department of Electrical and Computer Engineering in the College of Engineering and was also a Professor in the College of Optical Sciences. He was the Computational Electronics and Electromagnetics Thrust Area Leader with the Engineering Research Division of the Lawrence Livermore National Laboratory before joining The University of Arizona, Tucson, AZ, USA, in 1990. His current research interests include the application of new mathematical and numerical methods to linear and nonlinear problems dealing with the interaction of electromagnetic and acoustic waves with complex linear and nonlinear media, as well as metamaterials, metamaterial-inspired structures, nano-structures, and other classical and quantum applications-specific configurations. 